Kooperationsgemeinschaft Mammographie

\section{Neue Evaluation zum Brustkrebs-Screening}

Die „Kooperationsgemeinschaft Mammographie" hat einen aktuellen Bericht zur Ergebnis- und Prozessqualität im deutschen Brustkrebs-Screeningprogramm vorgelegt. Im Berichtszeitraum 2012 wurden rund 4,9 Millionen Frauen eingeladen, 2,7 Millionen nahmen teil. Entdeckt wurden dabei 17.311 Mammakarzinom.

Der positive Vorhersagewert lag bei 13\%. Von 100 Frauen mit verdächtigem Befund haben demnach 13 tatsächlich Krebs. Nach weitergehender nicht invasiver Diagnostik (mammografische Zusatzaufnahmen, Ultraschall, evtl. Magnetresonanz-Mammo- grafie) steigt der Vorhersagewert auf 50\% - eine von zwei Frauen, bei denen der Verdacht auch nach der Abklärung bestehen bleibt, hat demnach Krebs, die andere nicht. Die Prozessqualität des Screeningprogramms war gut. Die von den EU-Leitlinien vorgegebenen Mindestanforderungen bzw. Empfehlungen werden erreicht. Beispielsweise liegt die Bildwiederholungsrate bei $1,1 \%$ (Mindestanforderung $<3 \%$, Empfehlung < 1\%).

- Kooperationsgemeinschaft Mammographie (Hg.) Evaluationsbericht 2005-2012 - Ergebnis- und Prozessqualität im deutschen Mammographie-Screening-Programm. Berlin, August 2015

\title{
H.-pylori-negative Trinker
}

\section{Erhöhtes Risiko für Magenkrebs durch Alkohol}

Alkohol fördert die Entstehung von Magenkrebs. Der Effekt scheint sich aber nur bei Menschen bemerkbar zu machen, die nicht mit H. pylori infiziert sind. In einer Kohorte mit 18.863 Koreanern, darunter 403 Magenkarzinompatienten, war das Risiko für den Krebs signifikant erhöht, wenn Alkohol seit mehr als 30 Jahren in höheren
Mengen konsumiert wurde. Allerdings traf dies nur für Teilnehmer zu, die keine H.pylori-spezifischen IgG-Antikörper aufwiesen: Bei mindestens sieben alkoholischen Getränken pro Woche war das Risiko rund 3,5-mal und bei Binge-Drinking rund 3,3-mal so hoch wie bei Abstinenz. BJC 2015; online 17. September. doi: 10.1038/bjc.2015.333
Dr. med.

Brigitte Moreano Stellvertretende Chefredakteurin brigitte.moreano@ springer.com

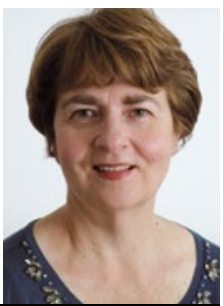

Risikomarker

Nächtlicher Hochdruck: Droht ein Diabetes?

Wenn der Blutdruck in der Nacht nicht absinkt, kann das, einer spanischen Studie zufolge, ein früher Hinweis darauf sein, dass sich ein Typ-2-Diabetes entwickelt.

An der prospektiven Untersuchung nahmen 2.656 Nicht-Diabetiker (Durchschnittsalter 50,6 Jahre) teil. Der Blutdruck wurde mittels 48-Stunden-Langzeitmessung (ABDM) ermittelt. Während einer mittleren Beobachtungszeit von 5,9 Jahren entwickelten 190 Personen einen Diabetes. Nach Berücksichtigung anderer Risikofaktoren, wie Taillenumfang, Nüchternglukose und Nierenerkrankungen, erschienen die nächtlichen Blutdruckwerte als zuverlässigster Prädiktor für eine Diabetes-Neuerkrankung: Pro Anstieg um eine Standardabweichung (SD) erhöhte sich das relative Risiko um 28\%. Überdies hatten hypertensive Patienten, die mindestens ein blutdrucksenkendes Medikament vor dem Schlafengehen einnahmen, ein signifikant niedrigeres Diabetesrisiko als diejenigen, die ihre Antihypertensiva über den Tag verteilten (HR 0,43).

- Diabetologia 2015, online 23. September; doi: 10.1007/s00125 015-3748-8

\section{Verletzungsrisiken bei den Kleinsten Nicht ohne Helm aufs Dreirad!}

Kopf und Ellenbogen sind beim Dreiradfahren besonders gefährdet und sollten entsprechend geschützt werden. In den Jahren 2012 und 2013 wurden in den USA 9.340 Kinder mit Dreiradunfällen behandelt, $2,4 \%$ davon stationär. Fleischwunden machten insgesamt 28,2\% aller Verletzungen aus und standen in der Gruppe der Einjährigen mit 27,9\% an erster Stelle. Bei den Drei- bis Fünfjährigen lagen Verletzungen innerer Organe an der Spitze (29,9-32,9\%) und bei Siebenjährigen kam es am häufigsten zu Prellungen (70\%). Das am stärksten gefährdete Körperteil und der häufigste Ort innerer Verletzungen war der Kopf (29,6\% bzw. 99,6\%). Von Frakturen waren mit 47,4\% am häufigsten die Ellenbogen betroffen. 